\title{
Validação de face e construto do Instrumento de Avaliação de Redes de Atenção Materno-infantil (IARAMI)
}

\author{
Face and construct validation of the Instrument of Evaluation \\ of Maternal and Child Care Networks (IEMCN)
}

Carolina de Oliveira Azim Schiller (https://orcid.org/0000-0001-5593-4191) ${ }^{1}$

William Augusto Gomes de Oliveira Bellani (http://orcid.org/0000-0002-1429-616X) ${ }^{2}$

Samuel Jorge Moysés (https://orcid.org/0000-0003-3075-6397) ${ }^{3}$

Renata Iani Werneck (https://orcid.org/0000-0003-1134-5357) ${ }^{3}$

Sérgio Aparecido Ignácio (https://orcid.org/0000-0002-8242-3781) ${ }^{3}$

Simone Tetu Moysés ( https://orcid.org/0000-0003-4861-9980) ${ }^{3}$

${ }^{1}$ Secretaria de Estado da Saúde do Paraná. R. Piquiri 170, Rebouças. 80230140 Curitiba PR Brasil. caroazim@hotmail.com ${ }^{2}$ Departamento de Medicina, Faculdades Pequeno Príncipe. Curitiba PR Brasil.

${ }^{3}$ Escola de Ciências da Vida, Pontifícia Universidade Católica do Paraná. Curitiba PR Brasil.

\begin{abstract}
The organization of health systems in Health Care Networks (HCN) presents itself as a strategy to overcome the fragmentation of maternal and child health actions and services and to qualify care management. The objective of this study was to validate the Instrument for Evaluation of Maternal and Child Health Care Networks (IARAMI), adapted from the Evaluation Tool of Health Care Networks. The validation process comprised face and construct validation. The methodology used for face validation included the conceptual and semantic adaptation of the instrument and analysis by a panel of experts, through the modified consensus e-Delphi technique. After face validation, IARAMI was applied in a sample of 99 health managers of municipalities in the state of Paraná. The construct validation was conducted through the internal consistency analysis using Cronbach's alpha coefficient and factorial analysis. The factorial analysis technique was applied to each of the dimensions of the instrument, enabling the analysis of communalities. The results showed that IARAMI presented reliability and validity, proving to be a tool that can help managers and health workers in the planning, management, and evaluation of the degree of integration of the maternal and child care network.
\end{abstract}

Key words Health systems, Health care, Health evaluation
Resumo A organização dos sistemas de saúde em redes de atenção à saúde (RAS) se apresenta como uma estratégia para superar a fragmentação das ações e serviços de saúde materno-infantil e qualificar a gestão do cuidado. O objetivo deste estudo foi validar o Instrumento de Avaliação de Redes de Atenção Materno-infantil (IARAMI), adaptado a partir do Instrumento de Avaliação de Redes de Atenção à Saúde. O processo de validação compreendeu a validação de face e construto. A metodologia utilizada para a validação de face incluiu a adaptação conceitual e semântica do instrumento e a análise por um painel de especialistas, por meio da técnica e-Delphi de consenso modificada. Após sua validação de face, o IARAMI foi aplicado em uma amostra de gestores de 99 municípios do estado do Paraná. A validação de construto foi conduzida por análise de consistência interna por meio do coeficiente alfa de Cronbach e de análise fatorial. A técnica de análise fatorial foi aplicada para cada uma das dimensões do instrumento, permitindo a análise de comunalidades. Os resultados evidenciaram que o IARAMI apresentou confiabilidade e validade, demonstrando ser uma ferramenta que pode auxiliar gestores e trabalhadores da saúde no planejamento, na gestão e na avaliação do grau de integração da rede de atenção à saúde materno-infantil.

Palavras-chave Sistemas de saúde, Atenção à saúde, Avaliação em saúde 


\section{Introdução}

Gestores e governantes, nos âmbitos nacional e internacional, têm priorizado as políticas voltadas para a qualificação da atenção à saúde materno-infantil e a organização de serviços de saúde efetivos voltados para esses grupos populacionais. Com o intuito de fomentar a melhoria nos indicadores globais de saúde materno-infantil, a Organização das Nações Unidas (ONU) incluiu nos objetivos para o desenvolvimento sustentável assegurar uma vida saudável e promover o bem - estar para todos, em todas as idades, por meio da redução, até 2030, da taxa de mortalidade materna e acabar com as mortes evitáveis de recém-nascidos e crianças menores de cinco anos ${ }^{1}$. Essa preocupação global se apoia nas evidências de que os cuidados nos primeiros anos irão impactar a saúde dos indivíduos ao longo da vida².

A organização dos serviços de saúde materno-infantil em redes se apresenta como uma estratégia para alcançar a atenção integral com qualidade e equidade e assim superar a fragmentação das ações em serviços de saúde e qualificar a gestão do cuidado. Seu objetivo é garantir acesso, acolhimento e resolutividade dos serviços, assegurando às mulheres o direito ao planejamento reprodutivo, a atenção humanizada na gravidez, no parto, abortamento e puerpério, e às crianças o direito ao nascimento seguro, ao crescimento e desenvolvimento saudáveis, com a consequente redução da mortalidade materna e neonatal ${ }^{3}$.

Integrar serviços de saúde com base nas necessidades, ao longo do tempo e por meio de diferentes níveis de atenção, tem sido preconizado por organizações internacionais ${ }^{4,5}$ e priorizado no Brasil como forma de garantir a integralidade do cuidado. ${ }^{6}$ As redes de atenção à saúde (RAS) representam, portanto, a forma de organização das ações e serviços de promoção, prevenção e recuperação da saúde, em todos os níveis de complexidade, de determinado território, de modo a permitir a articulação e a interconexão de conhecimentos, saberes, tecnologias, culturas, profissionais e organizações existentes, para que o cidadão possa acessá-los de acordo com suas necessidades de saúde, de forma racional, harmônica, sistêmica, regulada e conforme lógica técnicosanitária ${ }^{7}$. As RAS são consideradas instrumentos de garantia do direito à saúde, ampliando acesso e diminuindo desigualdades ${ }^{8}$.

Segundo Mendes ${ }^{9}$, a operacionalização das RAS depende da interação entre a população atendida, o modelo de atenção proposto e a estrutura operacional, que compreende a organiza- ção das atenções primária, secundária e terciária, os sistemas de apoio (diagnóstico e terapêutico, assistência farmacêutica e informação em saúde), sistemas logísticos (cartão de identificação das pessoas usuárias, prontuário clínico, acesso regulado à atenção e sistemas de transporte em saúde), além do sistema de governança.

A avaliação do processo de implementação das redes de atenção à saúde materno-infantil por um instrumento que avalie sistematicamente a complexidade e a integração da rede, e que seja válido e confiável, é essencial para apoiar gestores e profissionais da saúde na tomada de decisão para garantia desse cuidado. Até o momento, não há na literatura um instrumento com essas características.

O objetivo deste estudo foi validar o Instrumento de Avaliação de Redes de Atenção Materno-infantil (IARAMI), adaptado a partir do instrumento de avaliação de redes de atenção à saúde proposto por Mendes9. Este artigo apresenta o processo de validação de face e construto desse instrumento, que se propõe a avaliar o perfil de organização e a integração das redes de atenção à saúde materno-infantil.

\section{Material e método}

Trata-se de um estudo metodológico de validação do IARAMI, instrumento que se destina a fornecer aos gestores e profissionais de saúde um diagnóstico do perfil de integração em que se encontra a rede de atenção à saúde materno-infantil e a identificação da necessidade de melhoria da atenção à saúde nessa rede.

O processo de validação compreendeu validação de face e construto. A validação de face envolveu a participação de uma equipe multidisciplinar de pesquisadores e profissionais da área de saúde materno-infantil, para que fosse realizada a análise conceitual e semântica do instrumento -base ${ }^{9}$, possibilitando sua adequação para avaliação de redes de atenção à saúde materno-infantil.

$\mathrm{O}$ instrumento-base de avaliação de redes de atenção à saúde desenvolvido por Mendes ${ }^{9}$ contempla 174 questões, organizadas em sete dimensões teóricas, ou componentes: população, atenção primária, atenção secundária e terciária, sistema de apoio, sistema logístico, sistema de governança e modelo de atenção à saúde. Na aplicação do instrumento, o respondente atribui valores aos itens avaliados sobre cada componente, variando de 0 a 3 , em que: 0 - corresponde à não existência do item que está sendo perguntado; 1 - 
existe o que está sendo perguntado, mas com um funcionamento básico ou incipiente; 2 - existe o que está sendo perguntado, com um funcionamento razoavelmente bom, mas insuficiente; e 3 - corresponde à sua existência em operação de forma ótima. Após o cálculo do escore do instrumento pela soma dos valores atribuídos, o resultado pode ser interpretado como: de 0 a 130 - incapacidade para a operação da RAS, sistema fragmentado; de 131 a 260 - capacidade básica para operar a RAS, rede de atenção à saúde incipiente; de 261 a 390 - capacidade razoavelmente boa para operar a RAS, rede de atenção à saúde avançada; e de 391 a 522 - capacidade ótima para operar a RAS, redes integradas de atenção à saúde. Além dessa análise quantitativa, o instrumento original prevê uma análise qualitativa, selecionando-se os escores de cada questão inferiores a 3, mas especialmente os de valores 0 e 1 . Esses pontos críticos deverão estar presentes no plano de desenvolvimento institucional da rede de atenção à saúde. Os resultados desta avaliação possibilitam a identificação de áreas críticas de desempenho da mesma, apoiando gestores e trabalhadores de saúde a instituir estratégias que permitam fortalecer e qualifica-la para sua integração.

Com base nos procedimentos metodológicos proposto por Fernandes ${ }^{10}$, o processo de validação de face do IARAMI é descrito na Figura 1.

O processo de validação de face se iniciou com a adaptação preliminar do instrumento-base, realizada pelos pesquisadores para o contexto da Rede de Atenção Materno-Infantil. Após adaptação e consenso com o autor do instrumento-base, o novo instrumento passou a ter 152 questões, organizadas nas sete dimensões teóricas.

A etapa seguinte compreendeu a composição de um painel de 16 especialistas da área de atenção à saúde materno-infantil para a validação de face do IARAMI. A técnica de consenso e-Delphi modificada $^{11}$ foi utilizada para a busca de consenso nas opiniões dos especialistas a respeito dos indicadores ou itens propostos no instrumento. Após informações gerais sobre a pesquisa e o aceite de participação por meio de Termo de Consentimento Livre e Esclarecido, os especialistas responderam a um formulário on-line contendo, inicialmente, informações gerais para a caracterização do grupo (idade, sexo, formação profissional, instituição de vinculação e função na instituição). Os especialistas foram então convidados a analisar o instrumento a ser validado na sua primeira versão, conforme a importância de inclusão de cada item, atribuindo valores de 0 a 4, apresentados em uma escala Likert ( 0 - nada importante, não vejo a menor necessidade de incluir esse item; 1 - pouco importante, mas pode ser incluído; 2 - importante, mas se tiver que cortar alguma coisa, esta poderia ser excluída; 3- muito importante, seria muito bom incluir; 4 - essencial, deve ser incluído, obrigatoriamente). Os especialistas ainda podiam apresentar sugestões de alteração ou inclusão de novas perguntas.

A consulta aos especialistas foi realizada em duas rodadas. Em cada uma, o consenso foi confirmado pela estabilização das questões, considerando $70 \%$ de concordância sobre a importância de cada uma delas no instrumento. Foram mantidas ou estabilizadas as questões consideradas pelo painel de especialistas como muito importantes e essenciais. Questões não estabilizadas na primeira rodada foram reavaliadas em uma segunda rodada. As questões que tiveram sugestão de alteração na escrita foram submetidas a uma segunda rodada para análise dos especialistas, para que escolhessem entre a versão original ou a versão reformulada.

Após exclusões, adaptações e inclusões sugeridas pelos especialistas, foi obtida uma versão final do instrumento, composta por 131 questões organizadas nas sete dimensões teóricas do instrumento original, que devem ser avaliadas pelos respondentes conforme seu perfil de implementação. Um escore total obtido pela somatória das respostas permite verificar o perfil de integração da rede de atenção à saúde materno-infantil, sendo caracterizada como rede fragmentada, em estágio inicial, em estágio avançado ou rede integrada.

Após validação de face do IARAMI, o instrumento foi aplicado em uma amostra estratificada proporcional de respondentes gestores de $136 \mathrm{mu}$ nicípios do estado do Paraná. O sorteio dos municípios foi realizado de forma sistemática e ordenada por macrorregiões, segundo o nível de gestão (municipal e estadual) $^{12}$, o porte populacional (pequeno, médio e grande) ${ }^{13}$ e tamanho da população ${ }^{12}$. O instrumento foi disponibilizado em uma plataforma on-line e enviado por e-mail aos gestores municipais (secretários de saúde ou pessoa indicada com conhecimento sobre a organização e o funcionamento da rede de atenção materno-infantil). Todos os participantes concordaram com o Termo de Consentimento Livre e Esclarecido após terem sido informados acerca do estudo.

A validação de construto do IARAMI foi realizada a partir das respostas obtidas de uma amostra de 99 respondentes dos municípios que 


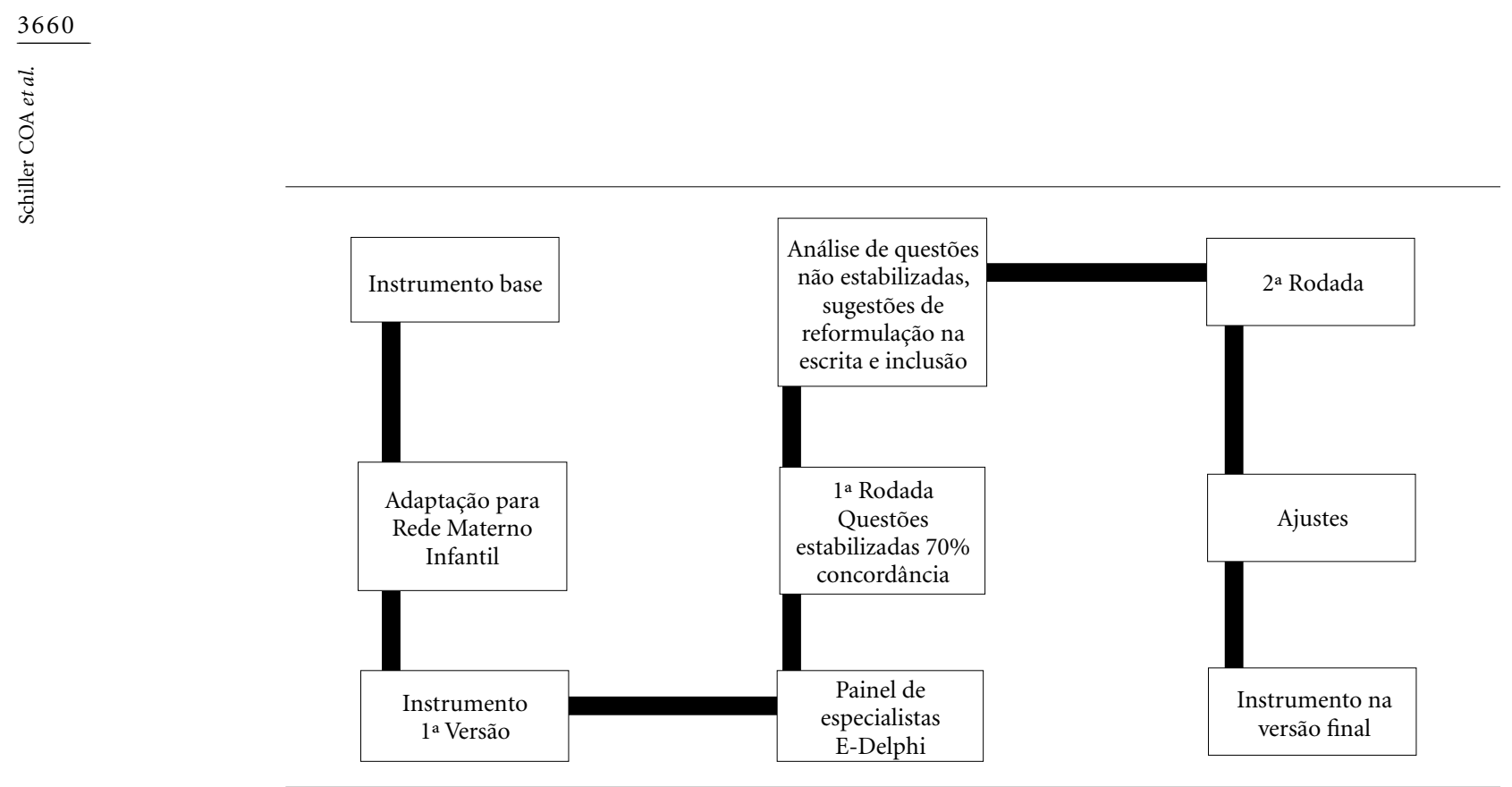

Figura 1. Etapas da validação de face do IARAMI.

Fonte: Elaborado pelos autores.

aceitaram participar do estudo. A validação da consistência interna do instrumento se deu por meio do coeficiente alfa de Cronbach, considerando valores aceitáveis aqueles acima de $0,7^{14,15}$.

Uma matriz de correlação entre os itens do instrumento foi utilizada para avaliar colinearidades. Itens que apresentaram colinearidade (coeficientes de correlação maiores que 0,7 ) foram reavaliados, com o objetivo de remover as questões que poderiam estar medindo conteúdos similares e assim tornar o instrumento mais conciso e efetivo. Essa reavaliação de cada item foi conduzida por quatro profissionais envolvidos com redes de atenção materno-infantil. Após a remoção das questões com alta colinearidade e consideradas dispensáveis pelo grupo de especialistas, um novo alfa de Cronbach foi calculado para confirmar a consistência interna do instrumento.

Para a análise da importância dos itens dentro de cada dimensão, utilizou-se o coeficiente alfa de Cronbach e a técnica de análise fatorial com método de rotação ortogonal Varimax, a fim de agrupar as variáveis (itens) em um número menor de fatores, que contivessem aproximadamente o mesmo total de informação expresso pelas variáveis originais, em função das interrelações entre as mesmas.

A análise da comunalidade, empreendida pela técnica de análise fatorial, ajudou a definir os itens importantes a permanecerem na dimensão, considerando a variância comum acima de
$50 \%$ com os demais itens ${ }^{14}$. Os itens com comunalidade abaixo desse patamar foram excluídos e a análise fatorial foi realizada novamente.

Os testes de Kaiser-Meyer-Olklin (KMO) e de esfericidade de Bartlett foram realizados para cada dimensão, a fim de verificar a adequação da análise fatorial. Para o teste de Kaiser-Meyer -Olklin foram considerados aceitáveis valores entre 0,5 a 1 , e o teste de esfericidade de Bartlett deveria ser estatisticamente significante $(\mathrm{p}<0,05)^{15}$.

Este estudo obteve aprovação do Comitê de Ética e Pesquisa da Pontifícia Universidade Católica do Paraná, da Secretaria de Estado da Saúde do Paraná - Hospital do Trabalhador e da Secretaria Municipal de Saúde de Curitiba.

\section{Resultados}

A validação de face do instrumento ocorreu em junho de 2014, compreendendo duas rodadas de consultas aos especialistas, com intervalo de 15 dias entre elas.

Das 152 questões contidas na primeira versão do instrumento, 38 (25\%) não foram estabilizadas na primeira rodada e oito $(5,26 \%)$ tiveram sugestão de reformulação na escrita. Essas questões foram reavaliadas na segunda rodada pelo painel de especialistas. Das oito que tiveram sugestão de alteração da escrita, duas, apesar de terem sido estabilizadas na primeira rodada, fo- 
ram eliminadas por abordarem conceitos específicos e complexos para serem compreendidos por gestores locais, tais como "a organização dos serviços pautada em princípios de economia de escala" e "integração horizontal por meio de fusões ou alianças estratégicas", "farmacoeconomia e farmacovigilância", e uma delas foi mantida na versão inicial. Ao final do processo, após as duas rodadas de análise pelo painel de especialistas, foram eliminadas 22 questões e uma questão foi incluída, totalizando 131 questões distribuídas nas sete dimensões teóricas originais. Além da remoção dos termos complexos, foi sugerido o esclarecimento de outros, como "fatores de risco proximais", "modelagem do ponto de atenção" e "determinantes sociais intermediários de saúde", para melhor compreensão do instrumento por parte dos gestores locais. Outras questões removidas por sugestão dos especialistas, por serem avaliadas como pouco ou nada importantes, parecem avaliar aspectos essenciais da rede de atenção, porém com um detalhamento desnecessário, como as questões relacionadas ao componente sistema de apoio, com perguntas referentes à assistência farmacêutica.

Os resultados do processo de validação de face com base no consenso do painel de especialistas, com a apresentação da versão inicial do instrumento, os resultados das $1^{\mathrm{a}} \mathrm{e} 2^{\mathrm{a}}$ rodadas de análise e a versão final ajustada podem ser acessados no link https://doi.org/10.48331/scielodata.7Z2IAT.

A coleta de dados, com a aplicação do instrumento na amostra de respondentes gestores de municípios, ocorreu em julho de 2014. Da amostra original de 136 municípios, 99 participaram do estudo, obtendo-se uma taxa de resposta de $72,8 \%$. Do total de respondentes, 85 eram do sexo feminino, 14 do masculino. A maioria (81) tinha formação profissional em enfermagem, os demais tinham formação em farmácia, odontologia, medicina, fisiologia, direito, serviço social, administração, história, filosofia, teologia, ensino fundamental e ensino médio.

A análise inicial da consistência interna do IARAMI resultou em um alto valor do coeficiente alfa de Cronbach $(\alpha=0,98)$. A matriz de correlação entre os 131 itens do instrumento demonstrou forte correlação $(r$ maior que 0,7$)$ entre 70 questões. Todas foram então reavaliadas por um grupo de especialistas, com o objetivo de eliminar aquelas que poderiam estar medindo conteúdos similares. Do total dessas questões, 18 foram retiradas e 52 mantidas. Dessa forma, o instrumento passou a ser composto por 113 perguntas. Um novo teste de confiabilidade de alfa de Cronba- ch foi conduzido após a retirada das questões e o valor do coeficiente demonstrou alteração pouco significativa $(\alpha=0,97)$.

A análise de confiabilidade pelo coeficiente alfa de Cronbach por dimensão demonstrou alta consistência interna nas sete dimensões do instrumento.

A análise fatorial por dimensão permitiu explorar a comunalidade entre os itens dentro de cada dimensão e assim identificar itens que pudessem ser retirados do instrumento. Do total, oito foram retirados da versão final por apresentarem variância comum abaixo de $50 \%$ em relação aos outros itens da dimensão. Sendo assim, o instrumento total passou a ter 105 itens, distribuídos nas sete dimensões teóricas propostas pelo instrumento original. Recalculado para esta versão final do instrumento, o coeficiente alfa de Cronbach não demonstrou alteração $(\alpha=0,97)$.

O Quadro 1 apresenta os resultados e estatísticas da validação de construto do IARAMI por dimensão.

A versão final do Instrumento de Avaliação de Redes de Atenção Materno-infantil (IARAMI), após validação de face e de construto, é apresentada no Quadro 2.

Para a interpretação dos resultados do instrumento foram atribuídos intervalos de escore utilizando distribuição quartílica a partir do escore total e dos escores por dimensão (Quadro 3). Dessa forma é possível avaliar a integração da rede de atenção materno-infantil quando se utiliza o instrumento total, assim como analisar a estrutura da rede por componente ou dimensão.

\section{Discussão}

A literatura a respeito dos métodos de avaliação de redes de atenção à saúde materno-infantil apresenta estudos sobre avaliações de programas ou de um ponto de atenção, não contemplando a avaliação da rede, seus elementos constitutivos, componentes e sua complexidade ${ }^{17-26}$. Apenas dois estudos exploram métodos de avaliação de redes de atenção materno-infantil. O estudo de Alidina e colaboradores ${ }^{27}$ examina a rede de serviços de saúde materna, neonatal e infantil a partir de um painel de bordo de avaliação do programa composto por sete critérios (cuidados adequados, acessibilidade, eficácia, satisfação, cuidados integrados e coordenados, responsabilidade e viabilidade) e estabelece 50 indicadores mensuráveis. $\mathrm{O}$ estudo utiliza entrevistas com usuários e dados do sistema de informação local. 


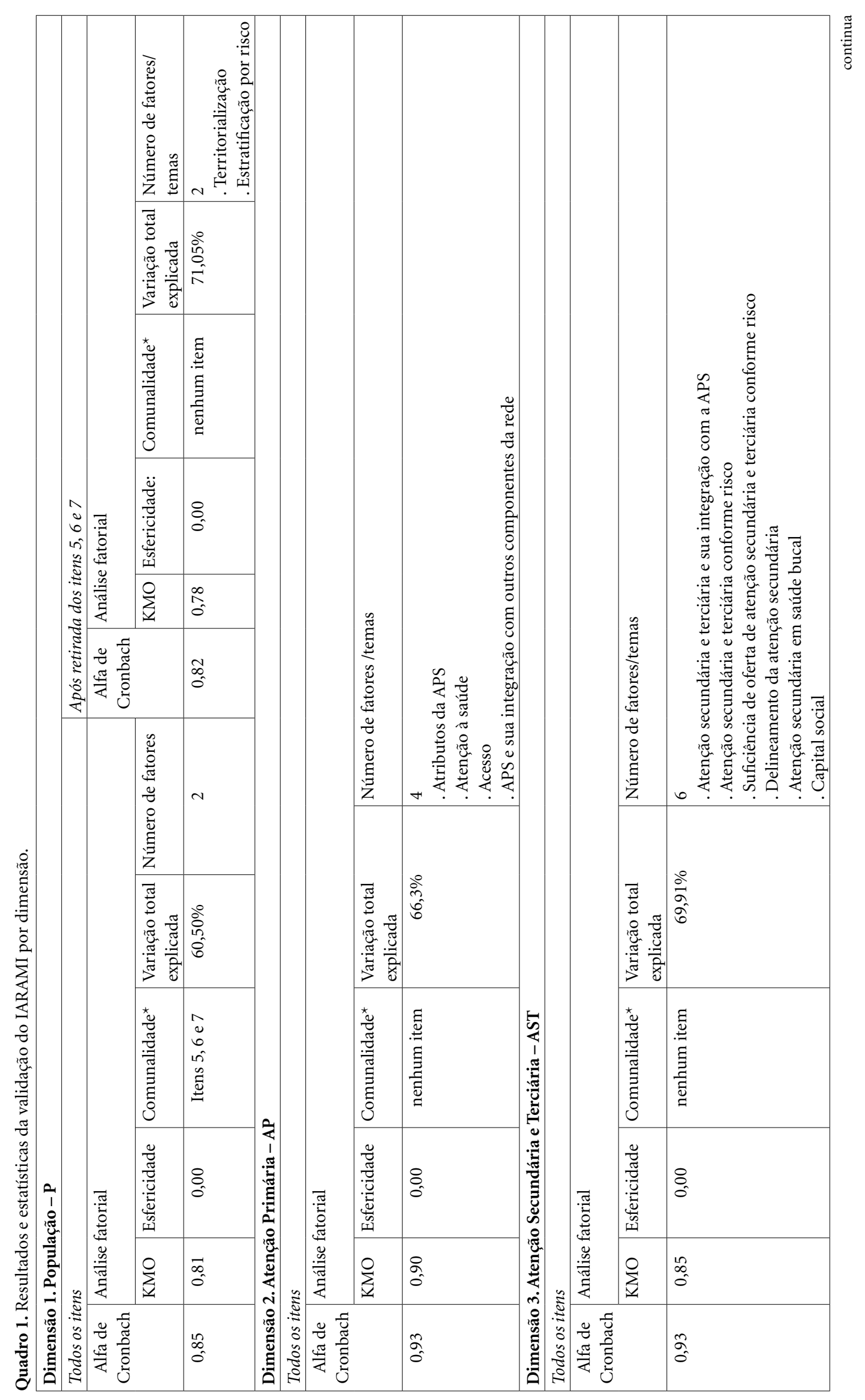




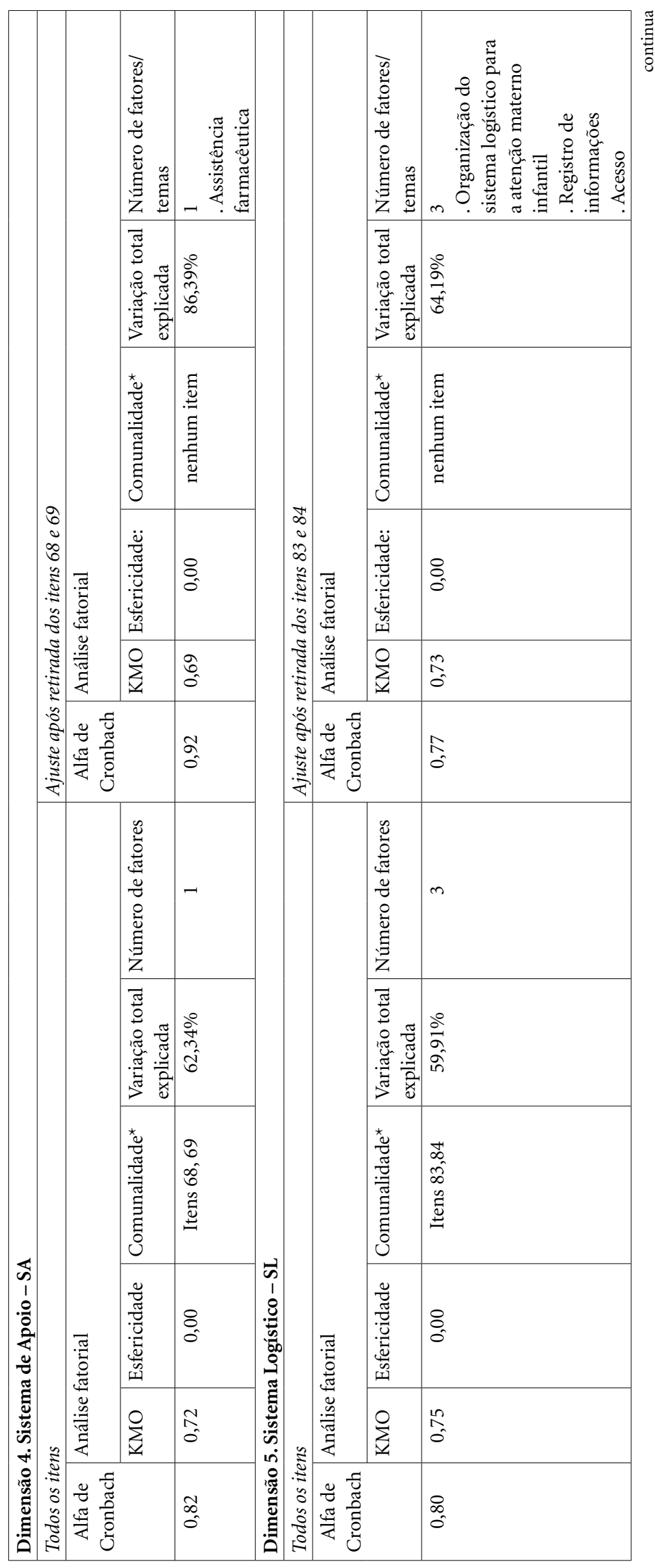




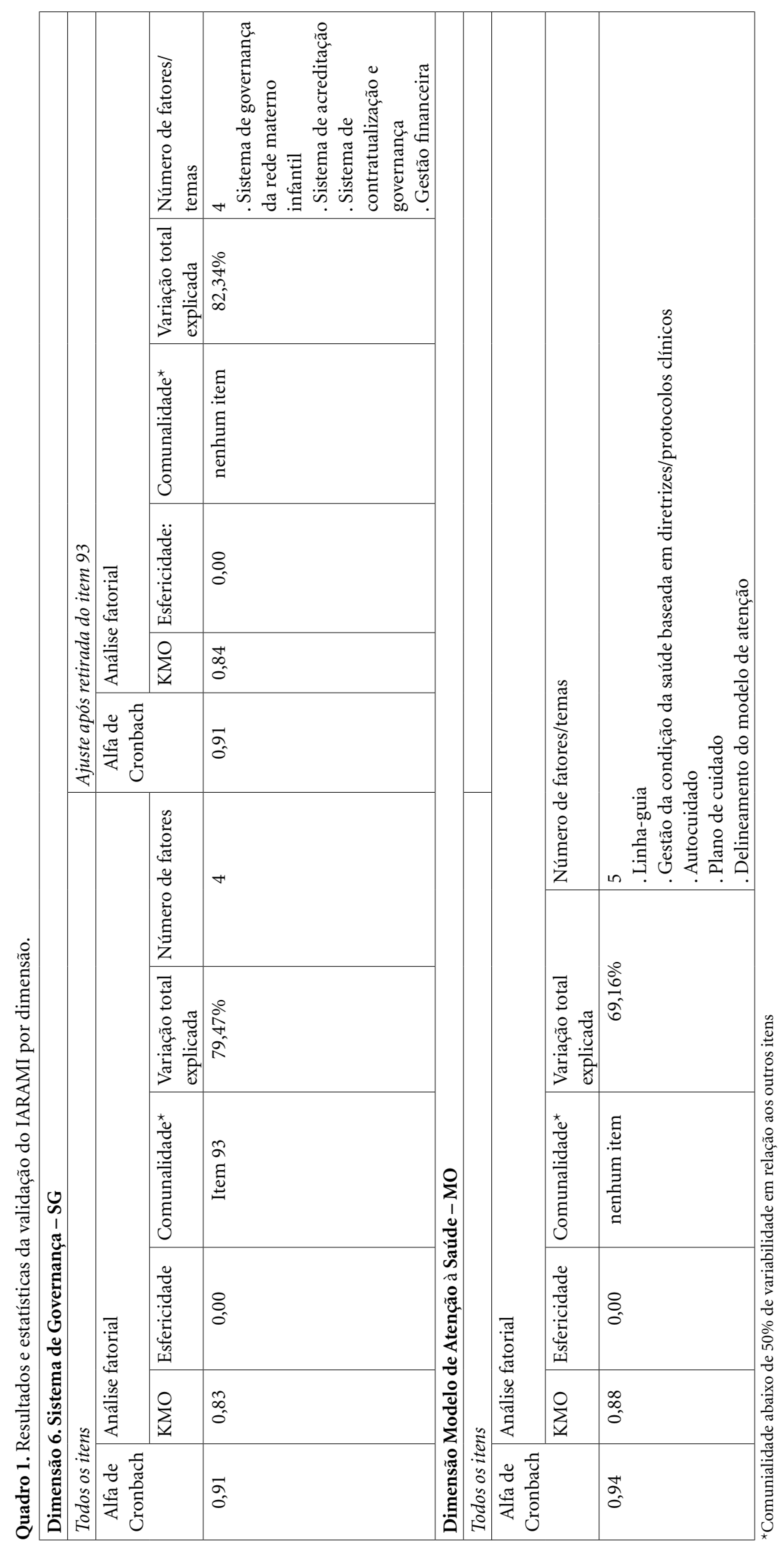


Quadro 2. Instrumento de Avaliação de Redes de Atenção à Saúde Materno-infantil (IARAMI) - versão final.

\section{População}

1. Há uma população claramente definida sob responsabilidade da rede de atenção materno-infantil (RMI)?

2. Há uma clara definição das necessidades de saúde desta população que compreende gestantes e crianças menores de dois anos?

3. A estrutura operacional da RMI é formatada em função dessas necessidades?

4. Há um processo de territorialização da RMI?

5. As gestantes e crianças menores de dois anos estão cadastradas na APS por unidade familiar?

6. As unidades familiares estão classificadas por riscos sociais?

7. A população está subdividida por fatores de risco proximais, aqueles relacionados a comportamentos e estilos de vida individuais?

\section{Atenção primária}

8. A equipe da APS tem claro o seu papel de responsabilização pelas gestantes e crianças menores de dois anos residentes no território?

9. Na APS há uma equipe multiprofissional, em que cada membro possui responsabilidades claras no cuidado das gestantes e crianças menores de dois anos?

10. Essas responsabilidades são orientadas pela diretriz/linha guia da RMI?

11. A APS é a porta de entrada para a RMI?

12. As relações entre APS e os demais níveis de atenção estão claramente definidos?

13. A APS opera com sistemas de informação suficientes para registrar e disponibilizar informações clínicas, epidemiológicas e gerenciais relativas às gestantes e crianças menores de dois anos (ex.: carteira da gestante, cartão da criança, Siab, Sispre-natal, etc.)?

14. Há uma boa interação entre as equipes da APS e de atenção especializada (consultas ou orientações rotineiras por escrito, por correio eletrônico ou sessões clínicas conjuntas)?

15. Há um vínculo estreito e contínuo entre as equipes da APS, as gestantes e suas famílias?

16. A APS está adequadamente organizada para prestar os cuidados às condições agudas e/ou aos eventos de agudização das condições crônicas de sua responsabilidade, de acordo com um sistema de classificação de risco das urgências e emergências e os protocolos clínicos?

17. A APS está adequadamente organizada para prestar os cuidados às gestantes e às crianças menores de dois anos, segundo a diretriz/linha-guia da RMI?

18. O prontuário clínico utilizado da APS está estruturado por família?

19. A APS realiza estratificação de risco das gestantes e crianças menores de dois anos, segundo a diretriz/linhaguia da RMI?

20. A APS realiza o plano de cuidado para cada gestante e criança menor de dois anos, conforme a diretriz linha-guia da RMI?

21. A APS monitora a adesão ao plano de cuidado de cada gestante e criança menor de dois anos?

22. A APS viabiliza o acesso da gestante de alto risco para a atenção ambulatorial secundária (pré-natal de alto risco), conforme diretriz/linha-guia da RMI?

23. A APS viabiliza o acesso da criança menor de dois anos de alto risco para a atenção ambulatorial secundária, conforme a diretriz/linha-guia da RMI?

24. A APS vincula a gestante de acordo com o grau de risco à maternidade de referência, conforme preconiza a diretriz/linha-guia da RMI?

25. As equipes da APS monitoram o comparecimento das gestantes de alto risco à atenção ambulatorial secundária?

26. As equipes de APS monitoram o comparecimento das crianças menores de dois anos de alto risco à atenção ambulatorial secundária?

27. As equipes da APS articulam-se rotineiramente com organizações comunitárias para a melhoria da atenção à gestante e criança?

continua

No outro estudo, Huçulak e colaboradores ${ }^{28}$ desenvolveram um painel de bordo para acompanhamento, monitoramento e avaliação da rede materno-infantil que estabeleceu indicadores nas seguintes perspectivas: resultado para a so- ciedade, de processo, de gestão e relacionados ao financiamento. Para dez objetivos estratégicos, foram relacionados 23 indicadores que possibilitam o acompanhamento da eficiência, efetividade e eficácia da rede ${ }^{28}$. Ambos os métodos per- 
Quadro 2. Instrumento de Avaliação de Redes de Atenção à Saúde Materno-infantil (IARAMI) - versão final.

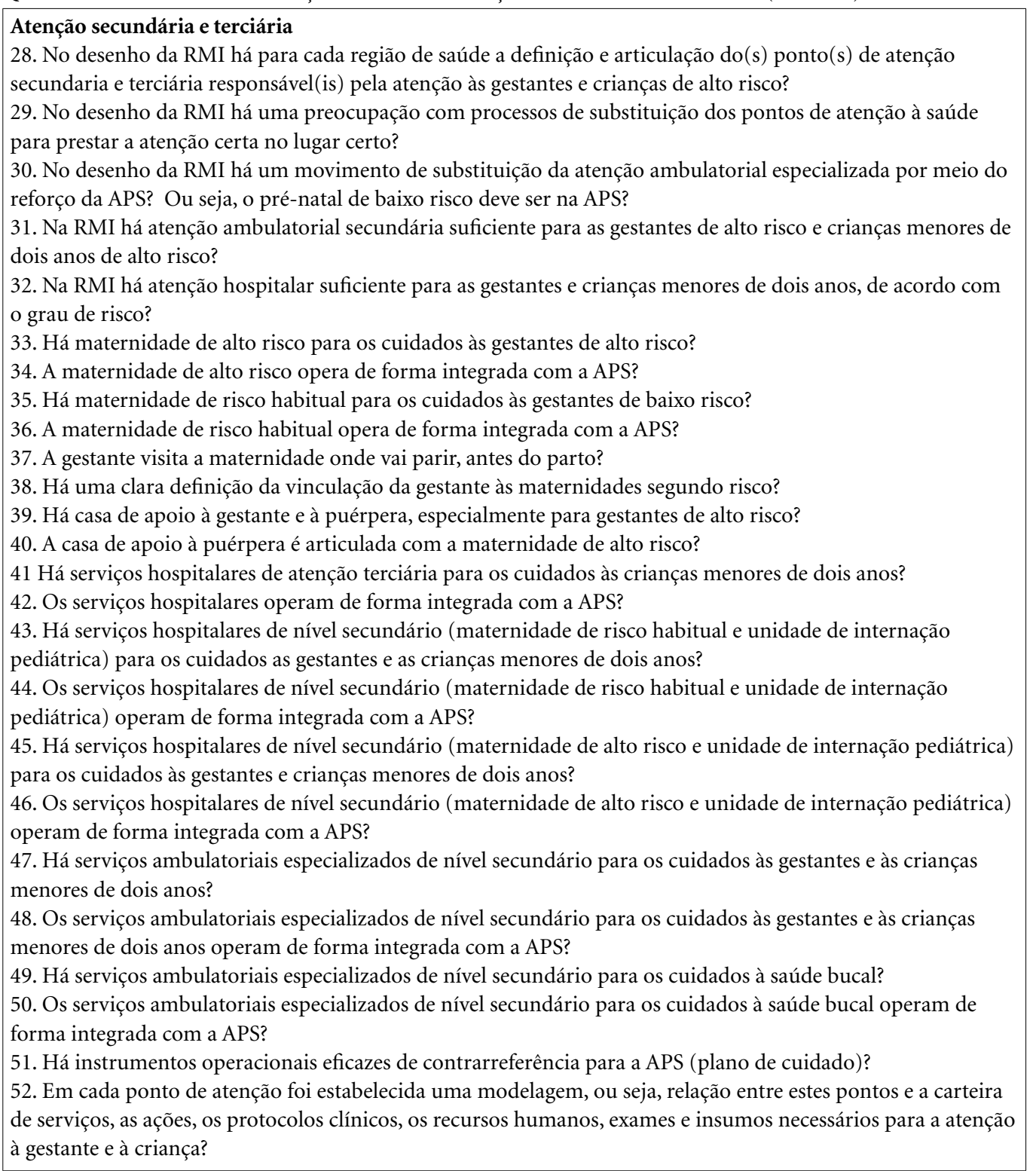

continua

mitem o acompanhamento, o monitoramento e a avaliação da rede de atenção à saúde materno -infantil por perspectivas distintas. No entanto, para a sua utilização, são necessários dados dos sistemas de informações disponíveis ou coleta de dados com usuários para mensurar a satisfação com os serviços e a atenção ofertada. Esses métodos requerem uma sistematização do processo de acompanhamento, monitoramento e avaliação, com uma maior organização dos serviços de saúde na coleta de dados e a manutenção do sistema de informação atualizado, o que pode representar inviabilidade em alguns contextos.

Já o instrumento de avaliação proposto neste estudo pode ser utilizado pelos gestores e trabalhadores da saúde sem necessidade de dados provenientes dos sistemas de informações ou de inquéritos populacionais, o que pode facilitar sua utilização. O IARAMI é um instrumento que deve ser usado para avaliar a gestão e a estrutura da rede, e não os resultados obtidos a partir disso. Pode ser aplicado a qualquer momento para ava- 
Quadro 2. Instrumento de Avaliação de Redes de Atenção à Saúde Materno-infantil (IARAMI) - versão final.

\begin{tabular}{|l|}
\hline Sistema de apoio \\
53. O sistema de assistência farmacêutica contempla a seleção dos medicamentos feita a partir da diretriz/ \\
linha-guia ou protocolos clínicos para as gestantes e crianças menores de dois anos? \\
54. O sistema de assistência farmacêutica contempla uma programação feita a partir das necessidades das \\
gestantes e crianças menores de dois anos adscritas à RMI? \\
55. O sistema de assistência farmacêutica contempla a atenção farmacêutica para as gestantes e crianças \\
menores de dois anos? \\
Sistema logístico
\end{tabular}

56. Existe um registro de base populacional único que integre as informações epidemiológicas e gerenciais relativas às gestantes e crianças menores de dois anos?

57. Há uma base de dados única que incorpore as gestantes e crianças menores de dois anos e as estratifica por grau de risco?

58. Há um cartão de identificação das gestantes e crianças menores de dois anos com um número único, articulado com a identificação da família e do território sanitário?

59. Há um prontuário familiar único que pode ser acessado nos diferentes pontos de atenção à saúde e nos sistemas de apoio da RMI?

60. A equipe da APS agenda diariamente as consultas de pré-natal de alto risco na atenção ambulatorial secundária?

61. A equipe da APS agenda diariamente alguns exames de análises clínicas ou de imagens?

62. Há carteira da gestante no município?

63. A carteira da gestante é utilizada rotineiramente?

64. Há carteira da criança no município?

65. A carteira da criança é utilizada rotineiramente?

66. O SAMU transporta gestante e crianças em situação de urgência e emergência?

67. O sistema tem um módulo de transporte de amostras de exames de análises clínicas?

\section{Sistema de governança}

68. Existe uma estrutura organizacional de governança da RMI?

69. A RMI tem um plano estratégico (mapa estratégico)?

70. O plano estratégico contempla um plano operativo da RMI de curto, médio e longo prazos?

71. A missão, a visão e os valores da RMI são claramente estabelecidos, conhecidos e compartilhados por todos os participantes?

72. Há um sistema de monitoramento e avaliação da RMI?

73. Há um sistema de acreditação em saúde da RMI na atenção primária?

74. Há um sistema de acreditação em saúde da RMI na atenção secundária?

75. Há um sistema de acreditação em saúde da RMI na atenção terciária?

76. Há um sistema de contratualização dos prestadores de serviços próprios (contratos internos) ou terceirizados (contratos externos)?

77. Esse sistema de contratualização está alinhado com os objetivos da RMI e contém incentivos para o alcance desses objetivos?

78. As gerências dos pontos de atenção à saúde e dos sistemas de apoio conhecem e aderem aos objetivos da RMI?

79. Os incentivos financeiros estão alinhados com os objetivos da RMI?

80. A RMI conta com mecanismos eficazes de gestão financeira?

continua

liar o grau de integração da rede, e em momentos distintos para avaliar se houve melhoria ou não na sua integração. Além disso, permite uma avaliação da estrutura da rede, pois analisa os itens por componente ou dimensão.

O IARAMI manteve a estrutura e o método de mensuração de modo similar ao instrumento-ba- se proposto por Mendes ${ }^{9}$ e se apresenta como um instrumento de fácil entendimento e aplicação.

Portanto, demonstrou ser uma ferramenta que pode auxiliar gestores e profissionais da saúde na avaliação do grau de integração da rede de atenção materno-infantil, apresentando um diagnóstico situacional, com suas fragilidades e 
Quadro 2. Instrumento de Avaliação de Redes de Atenção à Saúde Materno-infantil (IARAMI) - versão final.

Modelo de atenção

81. Há um modelo de atenção à saúde claramente definido e de conhecimento geral na APS e nos pontos de atenção à saúde?

82. O modelo de atenção à saúde articula subpopulações de risco com as intervenções em saúde?

83. O modelo de atenção à saúde propõe mudanças na organização do sistema de atenção à saúde?

Utilizam-se planos de autocuidado feitos em conjunto pela equipe de saúde e pelas gestantes usuárias da RMI?

84. Utilizam-se planos de autocuidado feitos em conjunto pela equipe de saúde e pelas gestantes usuárias da RMI?

85. A RMI contempla ações de autocuidado apoiadas por educação em saúde?

86. A RMI dispõe de diretriz/linha-guia para as condições de saúde para gestantes e crianças menores de dois anos?

87. A diretriz/linha-guia da RMI é comunicada adequadamente aos gestores e aos profissionais de saúde?

88. Essa diretriz/linha-guia organiza os fluxos e contrafluxos das pessoas na APS, nos pontos de atenção secundários e terciários e nos sistemas de apoio?

89. Essa diretriz/linha-guia estratifica por riscos as gestantes e crianças menores de dois anos com uma condição de saúde estabelecida?

90. No município existe uma programação do cuidado com o planejamento quantitativo por gestante e crianças menores de dois anos?

91. A diretriz/linha-guia da RMI é revisada periodicamente?

92. Essa diretriz/linha-guia é complementada por protocolos clínicos para utilização nos pontos de atenção secundários e terciários e nos sistemas de apoio?

93. O modelo de atenção inclui ações de promoção da saúde voltadas para os determinantes sociais de saúde de gestantes e crianças menores de dois anos?

94. O modelo de atenção à saúde inclui ações de prevenção das condições de saúde para gestantes e crianças menores de dois anos relacionados com fatores de risco proximais, comportamento e estilo de vida?

95. Há um plano de cuidado definido para cada gestante e criança menor de dois anos?

96. Esse plano de cuidado é avaliado e revisado periodicamente?

97. A gestão da condição de saúde envolve a educação permanente dos profissionais com base na diretriz/linhaguia ou nos protocolos?

98. A gestão da condição de saúde envolve uma programação realizada na APS, nos pontos de atenção secundários e terciários e nos sistemas de apoio com base na diretriz/linha-guia da RMI e protocolos?

99. A gestão da condição de saúde envolve um contrato de gestão com as equipes profissionais com base na programação?

100. A gestão da condição de saúde envolve a auditoria clínica realizada com base na diretriz/linha-guia e nos protocolos clínicos?

101. Os comitês fazem uma investigação rotineira dos óbitos infantis e maternos?

102. Essa auditoria clínica é realizada rotineiramente na APS e nos pontos de atenção à saúde secundários e terciários?

103. O modelo de atenção à saúde utiliza a tecnologia de gestão de caso para gestantes e crianças menores de dois anos com condições de saúde muito complexas?

104. A diretriz/linha-guia da RMI define as situações de saúde que devem ser manejadas pela gestão do caso? 105. Há um plano de cuidado elaborado para cada sujeito que está envolvido na gestão de caso?

Fonte: Elaborado pelos autores.

potencialidades. Desse modo, pode apoiar a criação de planos de intervenção que tenham como objetivo estruturar e organizar a rede de atenção à saúde materno-infantil para superar sua fragmentação. O instrumento também pode auxiliar gestores na elaboração de políticas públicas voltadas para a atenção materno-infantil e auxiliar trabalhadores da saúde na realização de boas práticas no cuidado da gestante e na puericultura.
Este estudo apresenta algumas limitações. Não foi possível a verificação da reprodutibilidade do instrumento. Isso se deu pela dificuldade de obtenção das respostas dos gestores, inviabilizando a reaplicação do instrumento ${ }^{29}$. Apesar disso, a satisfatória taxa de resposta possibilitou um adequado processo de validação de construto do instrumento, respondendo ao objetivo proposto pelo estudo. Na perspectiva de aperfei- 
Quadro 3. Interpretação dos resultados.

\begin{tabular}{|c|c|}
\hline \multicolumn{2}{|c|}{ IARAMI Escore total } \\
\hline 0 a 153 & $\begin{array}{l}\text { Incapacidade para a operação da rede } \\
\text { de atenção à saúde materno-infantil: } \\
\text { sistema fragmentado }\end{array}$ \\
\hline 154 a 196 & $\begin{array}{l}\text { Capacidade básica para operar a rede de } \\
\text { atenção à saúde materno-infantil: rede } \\
\text { de atenção incipiente }\end{array}$ \\
\hline 197 a 227 & $\begin{array}{l}\text { Capacidade razoavelmente boa para } \\
\text { operar a rede de atenção à saúde } \\
\text { materno-infantil: rede de atenção } \\
\text { avançada }\end{array}$ \\
\hline 228 a 298 & $\begin{array}{l}\text { Capacidade ótima para operar a rede de } \\
\text { atenção à saúde materno-infantil: rede } \\
\text { integrada de atenção }\end{array}$ \\
\hline \multicolumn{2}{|c|}{ Dimensão 1. População } \\
\hline 0 a 11 & Frágil/ruim \\
\hline 12 a 14 & Insuficiente \\
\hline 15 a 17 & Razoavelmente bom \\
\hline 18 a 21 & Ótimo \\
\hline \multicolumn{2}{|c|}{ Dimensão 2. Atenção primária } \\
\hline 0 a 35 & Frágil/ruim \\
\hline 36 a 43 & Insuficiente \\
\hline 44 a 51 & Razoavelmente bom \\
\hline 52 a 60 & Ótimo \\
\hline \multicolumn{2}{|c|}{ Dimensão 3. Atenção secundária e terciária } \\
\hline 0 a 30 & Frágil/ruim \\
\hline 31 a 40 & Insuficiente \\
\hline 41 a 49 & Razoavelmente bom \\
\hline 50 a 75 & Ótimo \\
\hline \multicolumn{2}{|c|}{ Dimensão 4. Sistema de apoio } \\
\hline 0 a 4 & Frágil/ruim \\
\hline 5 a 6 & Insuficiente \\
\hline 7 a 8 & Razoavelmente bom \\
\hline 9 & Ótimo \\
\hline \multicolumn{2}{|c|}{ Dimensão 5. Sistema logístico } \\
\hline 0 a 20 & Frágil/ruim \\
\hline 21 a 24 & Insuficiente \\
\hline 25 a 27 & Razoavelmente bom \\
\hline 28 a 35 & Ótimo \\
\hline \multicolumn{2}{|c|}{ Dimensão 6. Sistema de governança } \\
\hline 0 a 12 & Frágil/ruim \\
\hline 13 a 20 & Insuficiente \\
\hline 21 a 26 & Razoavelmente bom \\
\hline 27 a 39 & Ótimo \\
\hline \multicolumn{2}{|c|}{ Dimensão 7. Modelo de atenção à saúde } \\
\hline 0 a 35 & Frágil/ruim \\
\hline 36 a 44 & Insuficiente \\
\hline 45 a 55 & Razoavelmente bom \\
\hline 56 a 74 & Ótimo \\
\hline
\end{tabular}

Fonte: Elaborado pelos autores. çoar o instrumento, reforça-se a necessidade de estudos complementares, com a análise de outros parâmetros de validade.

Considerando que na literatura não havia um instrumento confiável e válido para avaliar as redes de atenção à saúde materno-infantil, o instrumento proposto pode auxiliar gestores e trabalhadores da saúde na melhoria da atenção à saúde materno-infantil.

\section{Colaboradores}

COA Schiller e ST Moysés participaram da concepção, do planejamento, da análise e interpretação da pesquisa, bem como da redação do artigo. SJ Moysés, RI Werneck, SA Ignácio e WAGO Bellani colaboraram na concepção, análise e interpretação do trabalho. 


\section{Referências}

1. Organização das Nações Unidas. Global Sustainable Development Report [Internet]; 2016. [acesso 2017 Out 10]. Disponível em: https://sustainabledevelopment.un.org/content/documents/2328Global\%20 Sustainable\%20development $\% 20$ report $\% 202016 \% 20$ (final).pdf

2. Victora CG, Aquino EM, Leal MC, Monteiro CA, Barros FC, Szwarcwald CL. Maternal and child health in Brazil: progress and challenges. Lancet 2011; 377(9780):1863-1876.

3. Brasil. Ministério da Saúde (MS). Rede Cegonha; 2011. [acesso 2017 Out 10]. Disponível em: https://bvsms.saude.gov.br/bvs/saudelegis/gm/2011/ prt1459_24_06_2011.html

4. Organización Panamericana de la Salud (OPS). Redes integradas de servicios de salud: conceptos, opciones de politica y hoja de ruta para su implementación en las Américas. Washington: OPS; 2010.

5. Organización Panamericana de la Salud. Redes integradas de servicios de salud basadas en la atención primaria. Washington: OPS; 2009.

6. Brasil. Ministério da Saúde (MS). Portaria no 4.279, de 30 de dezembro de 2010. Estabelece diretrizes para a organização da Rede de Atenção à Saúde no âmbito do Sistema Único de Saúde (SUS). Diário Oficial da União 2010; $31 \mathrm{dez}$.

7. Santos L, Andrade LOM. Redes no SUS: marco legal. In: Silva SF, organizador. Redes de atenção à saúde: desafios da regionalização no SUS. Campinas: Saberes; 2013. p. 22-34.

8. Kuschnir R, Chorny AH. Redes de atenção à saúde: contextualizando o debate. Cien Saude Colet 2010; 15(5):2307-2316.

9. Mendes EV. As redes de atenção à saúde. Brasília: Organização Pan-Americana da Saúde; 2011.

10. Fernandes LMAG. Validação de instrumento para avaliação de satisfação dos usuários com os serviços públicos de saúde bucal QASSaB [tese]. Camaragibe: Universidade de Pernambuco; 2002.

11. Giovinazzo RA. Modelo de aplicação da metodologia Delphi pela internet: vantagens e ressalvas. Administração online 2001; 2(2):1-11.

12. Paraná. Secretaria de Estado da Saúde. Plano Estadual de Saúde, 2016. Curitiba: Secretaria da Saúde; 2016.

13. Instituto Brasileiro de Geografia e Estatística (IBGE). Censo demográfico 2010 [Internet]. [acesso 2017 Out 10]. Disponível em: https://cidades.ibge.gov.br/brasil/ pr/panorama

14. Field A. Descobrindo a estatística usando o SPSS-2. Porto Alegre: Bookman; 2009.

15. Hair JF, Black WC, Babin BJ, Anderson RE, Tatham RL. Análise multivariada de dados. Porto Alegre: Bookman; 2009.

16. Brown TA. Confirmatory factor analysis for applied research. New York: The Guilford Press; 2006.

17. Silva ALA, Mendes ACG, Miranda GMD, Sá DA, Souza WV, Lyra TM. Avaliação da assistência hospitalar materna e neonatal: índice de completude da qualidade. Rev Saude Publica 2014; 48(4):682-691.

18. Taleb F, Perkins J, Ali NA, Capello C, Ali M, Santarelli C, Hoque DME. Transforming maternal and newborn health social norms and practices to increase utilization of health services in rural Bangladesh: a qualitative review. BMC Pregnancy Childbirth 2015; 15:75.
19. Yelland J, Riggs E, Szwarc J, Casey S, Dawson W, Vanpraag D, East C, Wallace E, Teale G, Harrison B, Petschel P, Furler J, Goldfeld S, Mensah F, Biro MA, Willey S, Cheng I-H, Small R, Brown S. Bridging the gap: using an interrupted time series design to evaluate systems reform addressing refugee maternal and child health inequalities. Implement Sci 2015; 10:62.

20. Ahmed S, Hossain A, Khan MA, Mridha MK, Alam A, Choudhury N. Using formative research to develop $\mathrm{MNCH}$ programme in urban slums in Bangladesh: experiences from MANOSHI, BRAC. BMC Public Health 2010; 10:663.

21. Basinga P, Gertler PJ, Binagwaho A, Soucat ALB, Sturdy J, Vermeersch MJ. Effect on maternal and child health services in Rwanda of payment to primary health-care providers for performance: an impact evaluation. Lancet 2011; 377(9775):1421-1428.

22. Schaffer MA, Goodhue A, Stennes K, Lanigan C. Populations at risk across the lifespan: program evaluations. Evaluation of a public health nurse visiting program for pregnant and parenting teens. Public Health Nurs 2012; 29(3):218-231.

23. Singh K, Akoto IO, Otchere F, Tettey SS, Barrington C, Huang C, Fordham C, Speizer I. Ghana's National Health insurance scheme and maternal and child health: a mixed methods study. BMC Health Serv Res 2015; 15:108.

24. Thomas SD, Hudgins JL, Sutherland DE, Ange BL, Mobley SC. Perinatal Program Evaluations: methods, impacts, and future goals. Matern Child Health J 2015; 19(7):1440-1446.

25. Holmes HA, Magoma M, John T, Levira F, Msemo G, Armstrong CE, Martínez-Álvarez M, Kerber K, Kihinga C, Makuwani A, Rusibamayila N, Hussein A, Lawn JE, Tanzanian Countdown Country Case Study Group. Tanzania's Countdown to 2015: an analysis of two decades of progress and gaps for reproductive, maternal, newborn, and child health, to inform priorities for post-2015. Lancet 2015; 3(7):396-409.

26. Zadoroznyj M. Postnatal care in the community: report of an evaluation of birthing women's assessments of a postnatal home-care programme. Health Soc Care Community 2015; 15(1):35-44.

27. Alidina S, Jordan M. The challenges of evaluating health systems networks: lessons learned from an early evaluation of the Child Health Network for the Greater Toronto Area. Healthc Manage Forum 2007; 20(2):22-27.

28. Huçulak MC, Peterlini OLG. Rede Mãe Paranaense: relato de experiência. Rev Esp Saude 2014; 15(1):7786.

29. Terwee CB, Bot SD, de Boer MR, van der Windt DA, Knol DL, Dekker J, Bouter LM, de Vet HC. Quality criteria were proposed for measurement properties of health status questionnaires. J Clin Epidemiol 2006; 60(1):34-42.

Artigo apresentado em 28/02/2019

Aprovado em 22/01/2020

Versão final apresentada em 24/01/2020

Editores-chefes: Romeu Gomes, Antônio Augusto Moura da Silva 